\title{
A Influência da Intensidade do Exercício Físico Aeróbio no Processo Aterosclerótico
}

\author{
The Aerobic Physical Exercise Intensity Influence on \\ the Atherosclerotic Process
}

Artigo de Revisão

\author{
Bruno Gonzaga Teodoro \\ Antônio José Natali' \\ Sílvio Anderson Toledo Fernandes' \\ Maria do Carmo Gouveia Peluzio' \\ 1. Universidade Federal de Viçosa, \\ MG, Brasil.
}

Endereço para correspondência: Maria do Carmo Gouveia Peluzio Universidade Federal de Viçosa. Av. PH Rolfs, Departamento de Nutrição e Saúde, s/n, Campus Universitário 36571-000 - Viçosa, MG, Brasil. E-mail:mpeluzio@ufv.br

\begin{abstract}
RESUMO
A aterosclerose é um processo inflamatório crônico e degenerativo que acomete os vasos, sendo caracterizada pelo acúmulo de lipídeos no espaço subendotelial da íntima, acúmulo de células inflamatórias e elementos fibrosos. A oxidação de LDL-c parece ser o principal evento para o início da aterosclerose. 0 exercício físico aeróbio melhora os sistemas de defesa orgânicos contra aterosclerose, diminuindo o estresse oxidativo e aumentando a síntese de enzimas antioxidantes; aumento da vasodilatação via óxido nítrico (NO) e óxido nítrico sintase endotelial (eNOS) e diminuição da inflamação sistêmica com produção de citocinas pró-inflamatórias e aumento de fatores anti-inflamatórios. Porém, de maneira aguda, o exercício aeróbio de alta intensidade aumenta o risco de desenvolvimento de eventos cardiovasculares e, de forma crônica, pode atuar negativa ou positivamente na prevenção do processo aterosclerótico.
\end{abstract}

Palavras-chave: Exercício Aeróbico, Aterosclerose, Intensidade do exercício

\begin{abstract}
Atherosclerosis is a chronic-degenerative inflammatory process that occurs in blood vessels and is characterized by accumulation of lipids, of inflammatory cells and fibrosis factors on the vessels walls. The (LDL-C) oxidation seems to be the main event to trigger atherosclerosis. Aerobic exercise improves the organic system defense against atherosclerosis by decreasing oxidative stress and increasing anti-oxidant enzyme biosynthesis, improving blood vessels vasodilatation by nitric oxide (NO) and endothelium nitric oxide synthase (eNOS), decreasing the pro-inflammatory cytokine production and increasing anti-inflammatory factors. However, acute high intensity aerobic exercises increase the cardiovascular event risk and its chronic type may affect either positively or negatively in the prevention of atherosclerosis.
\end{abstract}

Keywords: Aerobic exercise, atherosclerosis, exercise intensity

\section{INTRODUÇÃO}

As doenças cardiovasculares (DCV) são as principais causas de morbidade e mortalidade no Brasil e no mundo. Segundo dados do Instituto Brasileiro de Geografia e Estatística (IBGE), um terço de todas as mortes da população mundial (16,7 milhões de pessoas) foram ocasionadas pelas DCV ${ }^{(1)}$

O exercício físico regular está associado com um decréscimo na incidência de eventos cardiovasculares ${ }^{(2)}$. $O$ treinamento físico melhora a função endotelial, a capacidade física e o surgimento de vasos colaterais em pacientes com doenças arteriais coronarianas $(D A C)^{(3)}$, melhora a insuficiência cardíaca crônica ${ }^{(4)}$ e a doença arterial periférica ${ }^{(5)}$. Além disso, a atividade física está associada com melhora no peso corporal, pressão arterial, sensibilidade à insulina ${ }^{(2)}$, variáveis inflamatórias ${ }^{(6)}$ e hemostáticas ${ }^{(7)}$.

A intensidade pela qual se realiza atividade física aeróbica torna-se fator essencial para seus possíveis benefícios. Buscando esclarecer os pontos positivos e negativos a serem considerados em um acompanhamento de indivíduos saudáveis ou mesmo aqueles que necessitam de cuidados especiais frente à prática do exercício aeróbio, fez-se consulta à base de dados do Medline (PubMed) nos últimos 10 anos, utilizandose as palavras-chave aterosclerose e exercício aeróbio e suas respectivas traduções para o inglês; além disso, artigos com importância histórica e referências importantes encontradas nos artigos pesquisados também foram incluídos, para buscar evidências sobre a influência aguda e crônica da intensidade do treinamento aeróbio no processo envolvendo a doença cardiovascular.

\section{Gênese da aterosclerose}

A aterosclerose pode ser definida como um processo inflamatório crônico e degenerativo que acomete os vasos, sendo caracterizada pelo acúmulo de lipídeos no espaço subendotelial da íntima, acúmulo de células inflamatórias e elementos fibrosos. A aterosclerose pode se desenvolver em qualquer vaso, porém, os mais comumente afetados e de relevância clínica incluem a aorta e as artérias coronárias, carótidas e cerebrais ${ }^{(8)}$.

Uma das possíveis hipóteses para o início da aterosclerose é o acúmulo de partículas de lipoproteínas de baixa densidade (LDL) na 
matriz subendotelial da camada íntima das artérias. Este acúmulo será maior quanto maior for o nível de LDL circulante. O transporte da LDL através do endotélio com consequente retenção na íntima será mais eficiente nos locais onde a força de cisalhamento for maior, aumentando suscetibilidade para formação da lesão ${ }^{(8)}$. Emcontraste aos efeitos adversos de uma elevação da LDL, a concentração de lipoproteína de alta densidade (HDL) correlaciona-se inversamente com o desenvolvimento da aterosclerose ${ }^{(9)}$.

A LDL presente na corrente sanguínea difunde-se passivamente por diapedese através das células endoteliais, e sua retenção na parede do vaso parece envolver interações entre a apolipoproteína B, que faz parte da sua constituição, e as proteoglicanas, que estão presentes na camada íntima das artérias. Uma vez retida no espaço subendotelial, a LDL poderá ser quimicamente modificada, contribuindo, assim, para o processo inflamatório. A modificação química mais significante para o início da formação da lesão é a oxidação, com consequente formação da LDL oxidada (LDL-ox). A modificação oxidativa das partículas de LDL é resultado da ação de radicais livres e de enzimas, tais como mieloperoxidase, xantina oxidase, NADPH-oxidase, fosfolipases e outras lipases ${ }^{(8)}$. O estudo das interações da LDL oxidada depende da extensão da sua modificação, a qual pode variar de uma modificação mínima ( $\mathrm{mmLDL}$ ), em que as partículas de LDL ainda são reconhecidas pelos receptores nativos de $L D L$, até uma oxidação extensa, na qual a apolipoproteína B (apo-B) é fragmentada, e ao invés destas partículas serem reconhecidas pelos receptores nativos de LDL, serão identificadas pelos receptores scavenger expressos pelos macrófagos ${ }^{(10)}$.

A LDL-ox estimula a camada de células endoteliais a produzir moléculas de adesão celular como VCAM-1 (molécula-1 de adesão da célula vascular), ICAM-1 (molécula-1 de adesão intercelular), fatores de crescimento, tal como M-CSF (fator estimulador de colônia de macrófago) e proteínas quimiotáticas, como MCP-1 (proteína-1 quimiotática para monócitos), resultando na adesão e no recrutamento de monócitos e linfócitos circulantes para dentro da parede arterial|(8). Este processo está expresso de maneira resumida na figura 1.

Porém, no organismo humano existem linhas de defesa do processo aterosclerótico, dentre elas as enzimas antioxidantes, o óxido nítrico (NO) e o óxido nítrico sintetase endotelial (eNOS) e moléculas e citocinas anti-inflamatórias que estão envolvidas no processo gerador de aterosclerose.

O sistema antioxidante atua na atenuação das cargas de radicais livres no organismo, sendo importante na prevenção do processo aterosclerótico, já que este pode ser iniciado e agravado através da entrada no endotélio das lipoproteínas modificadas pelos radicais livres. Basicamente, há produção de três enzimas atuantes no processo antioxidante. São elas a superóxido dismutase (SOD), catalase (CAT) e glutationa peroxidase (GPX).

A SOD converte radical superóxido em peróxido de hidrogênio, e exerce papel fundamental na contenção da aterosclerose, pois a superexpressão desta enzima juntamente com a catalase, diminuiu a aterosclerose induzida por Benzo(a)pyrene em animais Knockout para apo-E $\mathrm{E}^{(11)}$. O aumento da SOD pode diminuir a aterosclerose por meio de coativação do relaxamento vascular induzido por $\mathrm{NO}$ mediado por e-NOS, pois a dismutação do radical $\mathrm{O}_{2}$, aumenta a biodisponibilidade de $\mathrm{NO}$ nas células endoteliais, já que em presença do radical $\mathrm{O}_{2}$. o NO é desviado para formação do peróxido nitrito ${ }^{(12)}$.A CAT reduz peróxido de hidrogênio a água. Yin et al. (2007) ${ }^{(13)}$ demonstraram que coexpressão da catalase é importante para que não haja elevação da proliferação de células musculares lisas, induzidas pela LDL-ox, pois foi demonstrado, por estes autores, que a espécie reativa $\mathrm{H}_{2} \mathrm{O}_{2}$ (peróxido de hidrogênio) aumenta a proliferação destas células, aumentando o processo aterosclerótico. Na presença da catalase esta proliferação não ocorreu, demonstrando a importância desta enzima para contenção do processo aterosclerótico. Além disso, em animais manipulados para a superexpressão de catalase, houve diminuição da aterosclerose induzida por Benzo(a)pyrene em animais Knockout para apo-E $\mathrm{E}^{(11)}$.

A GPX, assim como a CAT, reduz peróxido de hidrogênio a água, além de reduzir peróxidos lipídicos a alcoóis lipídicos. Baixas concentrações de GPX levam a detoxificação ineficiente dos peróxidos lipídicos e de hidrogênio, podendo favorecer a formação de radicais peroxila e hidroxila, respectivamente ${ }^{(14)}$. Lewis et al. ${ }^{(15)}$ demonstraram a importância da GPX na prevenção e contenção da aterosclerose, já que camundongos manipulados para não expressarem GPX aumentaram o processo de aterosclerose em relação aos camundongos controle e a falta de GPX foi relacionada com aumento de moléculas pró-inflamatórias tais como VCAM e aumento de macrófagos pró-inflamatórios. Além disso, camundongos apo-E-- manipulados para superexpressão de GPx diminuíram a evolução da aterosclerose, sendo essa redução justificada pela diminuição da peroxidação lipídica na parede arterial e diminuição da expressão das proteínas das moléculas de adesão VCAM e ICAM na aorta dos animais ${ }^{(16)}$.

A função endotelial vascular íntegra é essencial para manutenção da saúde das paredes dos vasos sanguíneos. O NO é um gás volátil, solúvel em lipídeos e produzido pelas células endoteliais pela ação da enzima eNOS a partir do aminoácido L-arginina ${ }^{(17)}$. Segundo Green et al.(17), o NO tem como função principal a vasodilatação, aumentando o lúmen dos vasos sanguíneos e diminuindo a força de cisalhamento. Esta age no endotélio como resultado do fluxo sanguíneo e, quanto maior sua ação, mais prejudicial aos vasos sanguíneos.

Além disso, a eNOS está envolvida em processo de neoangiogênese, formação de novos vasos sanguíneos. Em estudo feito por Laufs et al. ${ }^{(19)}$, animais Knockout para eNOS não apresentaram produção endotelial de células progenitoras endoteliais (EPC) circulantes, células estas responsáveis pela neoangiogênese em animais e humanos.

O resumo deste processo está representado na figura 1.

\section{Exercício aeróbio no processo aterosclerótico}

Estudos observacionais sugerem que a inatividade física e o baixo condicionamento cardiorrespiratório são fatores preditores de doença cardiovascular aterosclerótica ${ }^{(20,21)}$, e que o aumento da atividade física habitual e condicionamento cardiorrespiratório ${ }^{(22)}$ estão associados ao decréscimo em todas as causas de mortalidade em homens com acometimentos cardiovasculares pré-existentes.

A prática regular de exercício aeróbio tem sido utilizada e recomendada tanto para prevenção quanto para tratamento da aterosclerose. Assim, a Sociedade Brasileira de Cardiologia (SBC) ${ }^{(23)}$ recomenda a prática regular de exercício aeróbio, em uma frequência semanal de três a seis vezes, com duração de 30 a 60 minutos por sessão e em intensidade moderada (60 a 60\% da frequência cardíaca máxima) para prevenção e tratamento da aterosclerose e dislipidemias.

Em um estudo clássico, Siscovick et al. ${ }^{(24)}$ demonstraram, pela primeira vez, que o exercício físico vigoroso aumenta o risco coronariano primário durante a sessão do exercício. Em concordância com Siscovick et al.(24) outro estudo ${ }^{(25)}$ demonstrou que uma única sessão de exercício aeróbio vigoroso (30 minutos a $70 \%$ do $\mathrm{VO}_{2 \max }$ ) aumenta a tendência do desenvolvimento de placas trombóticas em voluntários saudáveis e sedentários, ao passo que a intensidade moderada ( $50 \%$ do $\mathrm{VO}_{2 \max }$ ) não provocou o mesmo efeito ao realizarem coleta do sangue antes e após a sessão e análise in vitro destas células.

No entanto, de maneira crônica, o exercício vigoroso pode diminuir a ocorrência de eventos cardiovasculares e está associado inversamente com a mortalidade(26), além de que, quanto maior a intensidade, maior a diminuição de fatores de risco associados com 


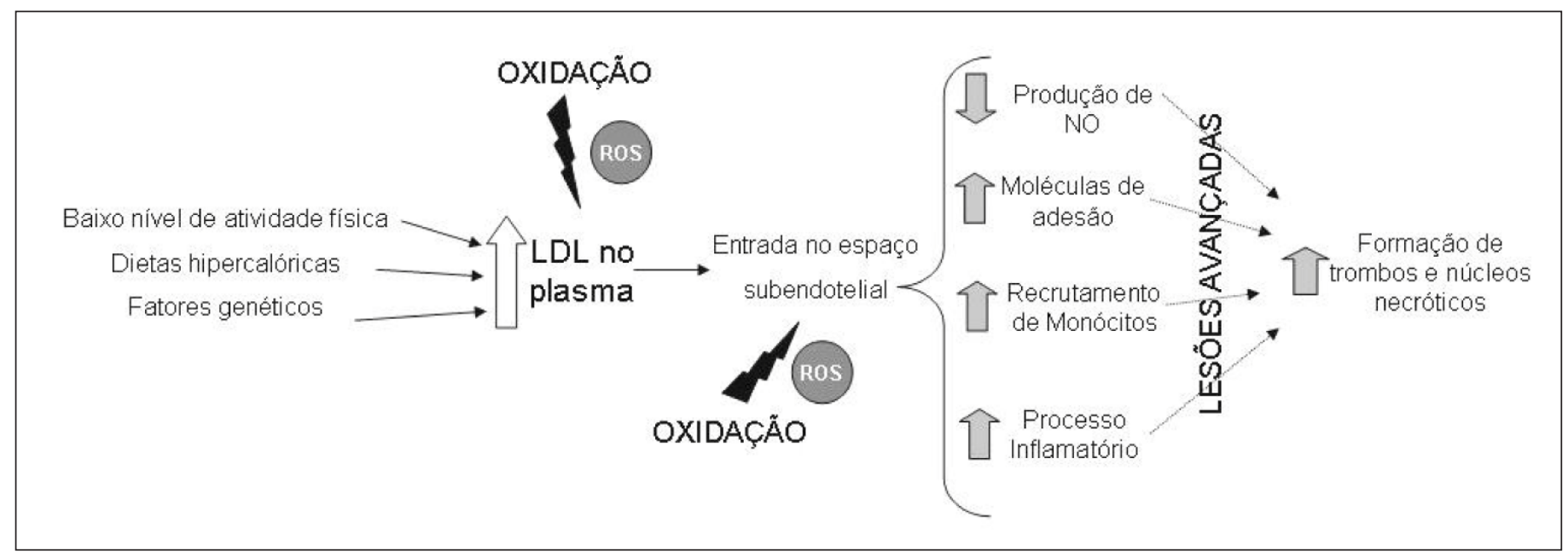

Figura 1. Diagrama do processo aterogênico.

a doença aterosclerótica, tais como a concentração plasmática de LDL-C e sobrepeso em adolescentes ${ }^{(27)}$.

Para verificar essas associações, Rauramaa et al. ${ }^{(28)}$ testaram, durante seis anos, a intervenção da atividade física aeróbica em um estudo randomizado e controlado (estudo DNASCO) em 140 homens de meiaidade na evolução da aterosclerose. Eles mensuraram a evolução da aterosclerose através da espessura da camada íntima arterial da bifurcação carótida por ultrassonografia. Os voluntários foram orientados a se exercitar em intensidade correspondente ao seu limiar ventilatório (de 40 a $60 \%$ do $\mathrm{VO}_{2 \max }$ ) de 45 a 60 minutos por sessão e cinco vezes por semana. Como principal achado do estudo, o grupo que sofreu intervenção e não fazia uso de estatina apresentou menor espessura da íntima arterial em relação ao grupo controle (sem exercício), o que demonstra que o exercício aeróbio de baixa a moderada intensidade representa fator protetor na evolução da lesão aterosclerótica.

Em estudos com animais, Shimada et al.(29) demonstraram que a atividade física aeróbica (natação), diminuiu a severidade da lesão aterosclerótica em camundongos Knockout para apo-E submetidos a uma dieta hiperlipídica. Além disso, Napoli et al. ${ }^{(30)}$ realizaram experimento com camundongos deficientes para o receptor de LDL e verificaram que a atividade física aeróbica progressiva (natação) além de atenuar a lesão aterosclerótica, aumenta o tempo de vida dos animais submetidos à dieta hiperlipídica. E ainda, Ramachandran et al.(31), demonstraram que o exercício aeróbio de moderada intensidade (corrida forçada em esteira, cinco vezes por semana, 30 minutos por dia a 15m/min) reduziu a lesão aterosclerótica quando comparado ao grupo controle (sem exercício), em camundongos Knockout para o receptor de LDL, previamente acometidos com aterosclerose, mostrando, assim, a possibilidade de o exercício não somente prevenir a aterosclerose como também reduzi-la.

Por outro lado, poucos demonstraram que o exercício físico não diminui a evolução do processo aterosclerótico como o de Williams et al. ${ }^{(32)}$, em que 34 meses de exercício aeróbio em macacos não impediram a progressão da lesão aterosclerótica, apesar de melhoria na função cardíaca, tais como diminuição da frequência cardíaca de repouso, melhora do volume de ejeção mensurado por ecocardiografia e na dilatação dos vasos em resposta à fenilefrina em relação aos animais que não se exercitaram.

Dessa maneira, o exercício aeróbio parece atuar na prevenção e tratamento da lesão aterosclerótica. A intensidade mais testada, tanto em humanos quanto em animais, é a moderada, havendo, porém, algumas evidências que exercícios aeróbios de intensidade alta, de maneira aguda, aumentam a chance de eventos cardiovasculares, mas cronicamente se associa com decréscimos na ocorrência desses eventos e da mortalidade.

\section{Potencial oxidante e antioxidante do exercício físico aeróbio}

Sabendo-se que uma das hipóteses mais aceitas para o início do processo aterogênico refere-se ao processo oxidativo envolvendo a $\mathrm{LDL}$, torna-se importante compreender os efeitos da atividade física aeróbia neste processo.

A maioria do oxigênio consumido é utilizado na mitocôndria para o metabolismo de substrato e produção de ATP. Porém, estima-se que para cada 25 moléculas de oxigênio consumidas durante a respiração normal, um radical livre é produzido ${ }^{(33)}$. Alguns estudos demonstraram que existe relação entre o aumento do consumo de oxigênio durante o exercício e a produção de radicais livres.

Em estudo de revisão, Pinho et al.(3) explicam que parte do oxigênio consumido pode ser convertido em vários intermediários, tais como $\mathrm{O}_{2}^{-}$(radical superóxido), $\mathrm{OH}^{-}$(radical hidroxila) e $\mathrm{H}_{2} \mathrm{O}_{2}$ (peróxido de hidrogênio). Por definição, apenas os dois primeiros são radicais livres, pois possuem um elétron não pareado em sua estrutura atômica. Coletivamente, eles são chamados de espécies reativas de oxigênio (ROS). Acredita-se que são responsáveis por uma série de mudanças bioquímicas e fisiológicas que ocorrem durante o exercício, sendo indicativos de estresse oxidativo, levando a danos nas estruturas celulares, como na bicamada lipídica das células, além da oxidação das lipoproteínas plasmáticas, principalmente a $L D L$, desencadeando o primeiro passo para gênese da aterosclerose ${ }^{(10)}$.

Em um estudo em que 10 homens saudáveis e bem condicionados exercitaram-se aerobicamente em intensidade leve (caminhada 55\% da frequência cardíaca de reserva), sem exaustão, durante seis horas e por dois dias consecutivos, demonstrou-se que houve diminuição da concentração plasmática da LDL oxidada ${ }^{(34)}$, evidenciando que o exercício físico de intensidade leve e longa duração poderá suprimir as cargas de estresse oxidativo de maneira aguda, podendo também se relacionar inversamente com o processo aterogênico.

Para verificar o efeito da intensidade do exercício físico aeróbio no estresse oxidativo, Wang et al. ${ }^{(35)}$ testaram o efeito de três protocolos de atividade física, leve $\left(40 \%\right.$ do $\left.\mathrm{VO}_{2 \max }\right)$, moderado $\left(60 \%\right.$ do $\left.\mathrm{VO}_{2 \max }\right)$ e intenso ( $80 \%$ do $\mathrm{VO}_{2 \max }$ ) por 40 minutos, sobre o aumento da LDL oxidada e ROS pós-exercício, em 25 adultos jovens e sedentários $\left(V_{2} O_{\text {max }}=35 \mathrm{ml} \cdot \mathrm{kg}^{-1} \cdot \mathrm{min}^{-1}\right)$. Verificou-se que, quando os voluntários se exercitaram na intensidade de $80 \%$ do $\mathrm{VO}_{2 \text { max }}$ demonstraram maior produção de LDL oxidada e que esta provocou maior reatividade das 
ROS nos monócitos, em relação às intensidades leve e moderada. Assim, conclui-se que, de maneira aguda, o exercício físico aeróbio de elevada intensidade provoca maior estresse oxidativo em indivíduos previamente sedentários, porém ainda não se sabe o efeito crônico deste exercício no processo aterosclerótico.

Cronicamente, procura-se demonstrar que a atividade física aeróbica em intensidade de moderada a alta melhoraria a função antioxidante, aumentando a expressão e atividade das enzimas antioxidantes. De Moraes et al. ${ }^{(36)}$ demonstraram, em modelo animal, que os grupos exercitados (corrida em esteira forçada, de 70 a $80 \%$ do $\mathrm{VO}_{2 \text { max }} 60$ minutos por dia e cinco dias por semana) obtiveram maior expressão da enzima antioxidante SOD no tecido aórtico e mesentérico, em relação aos grupos sedentários. De maneira semeIhante, Napoli et al. ${ }^{(37)}$ mostraram em camundongos Knockout para o receptor de LDL que a atividade física aeróbica de intensidade leve a moderada (natação) por 18 semanas levou ao aumento da atividade das enzimas antioxidantes, SOD, CAT e GPX no tecido aórtico dos grupos exercitados em relação aos sedentários. Além disso, Silva et al. ${ }^{(38)}$, também demonstraram oito semanas em dois protocolos de exercício em esteira - um de intensidade moderada e outro declinado - aeróbio foram efetivos na regulação do estresse oxidativo, aumento a atividade de SOD e diminuindo o dano oxidativo mensurado por hidroperóxidos lipídicos e conteúdo de proteína carobil. Dessa maneira, a atividade física crônica tem demonstrado aumento do sistema antioxidante enzimático, possibilitando ao organismo combater o processo aterogênico na sua origem.

Cabe salientar, porém, que a atividade física não usual, intensa e extrema pode levar ao aumento do estresse oxidativo até mesmo em indivíduos treinados. Para verificar esta hipótese, um estudo mostrou em 31 soldados com alto condicionamento físico $\left(\mathrm{VO}_{2 \max }=65 \mathrm{ml}\right.$ de $\mathrm{O}_{2} \cdot \mathrm{kg}^{-1} \cdot \mathrm{min}^{-1}$ ) que, após marcha de $50 \mathrm{~km}$ com 30kg de sobrecarga, houve aumento do estresse oxidativo e danos celulares mensurados pelos marcadores: ácido úrico, ácido ascórbico e conteúdo de proteína carbonil, mesmo sendo os voluntários treinados por seis meses antes da marcha ${ }^{(39)}$.

A atividade física crônica pode levar a adaptações benéficas no sistema de defesa antioxidante, ressaltando, porém, que altas intensidades podem aumentar o estresse oxidativo de maneira aguda. Entretanto, o efeito crônico dessas atividades e atividades extenuantes sob a danificação celular induzida pelas ROS não está claro.

\section{Exercício físico aeróbio e o sistema vasodilatador}

O sistema vasodilatador consiste basicamente na vasodilatação provocada pelo NO derivado do endotélio, sintetizado pela eNOS e estimulado por neurotransmissores como acetilcolina (AcH). MeIhoras nesse sistema têm demonstrado diminuição dos fatores de risco associados com aterosclerose, tais como hipercolesterolemia, hipertensão e obesidade ${ }^{(17)}$.

O treinamento físico aeróbio de moderada intensidade melhora a função endotelial, diminui a aterosclerose e aumenta o número de vasos sanguíneos (neoangiogênese) em animais e humanos. 0 aumento dos vasos sanguíneos é dependente, pelo menos em parte, do NO, pois estudo em animais Knockout para eNOS apresentou menor elevação das EPCs pós-exercício, sugerindo que o NO está correlacionado inversamente com o processo aterogênico(19).

Indivíduos idosos com maior condicionamento cardiorrespiratório apresentaram melhor função endotelial vasodilatadora do que indivíduos da mesma idade com menor condicionamento cardiorrespiratório ${ }^{(40)}$. Este mesmo estudo mostrou que intervenção de três meses de exercícios aeróbios de intensidade moderada (75\% da FC máxima, cinco vezes por semana) em indivíduos idosos sedentários melhorou o relaxamento vascular.

A fim de verificar o efeito crônico da intensidade do treinamento aeróbio nas propriedades vasodilatadoras em humanos, Goto et al. ${ }^{(41)}$ testaram indivíduos saudáveis por 12 semanas de treinamento em três diferentes intensidades, leve ( $25 \%$ do $\left.\mathrm{VO}_{2 \max }\right)$, moderada ( $50 \%$ do $\left.\mathrm{VO}_{2 \max }\right)$ e alta $\left(75 \%\right.$ do $\left.\mathrm{VO}_{2 \max }\right)$ com duração de 30 minutos por sessão e frequência semanal de cinco vezes. Verificaram, ao final do período da intervenção, que a melhora na função vasodilatadora ocorreu no grupo que treinou em intensidade moderada.

Similarmente aos achados de Goto et al. ${ }^{(41)}$, Sun et al. ${ }^{(42)}$ demonstraram diminuição da função vasodilatadora em animais submetidos a um treinamento estressante (três horas por dia a 50\% da velocidade aeróbica máxima, cinco vezes por semana, durante seis semanas) em comparação aos animais submetidos a treinamento menos estressante (duas horas por dia a 50\% da velocidade aeróbica máxima, cinco vezes por semana, durante seis semanas), apesar do aumento da eNOS em ambos os grupos, sugerindo que a função vasodilatadora não é somente dependente da síntese endógena de NO.

Porém, em estudo recente de Haram et al. ${ }^{(43)}$, mostrou-se, em modelo animal, que o treinamento intervalado de alta intensidade (três minutos a $90 \%$ do $\mathrm{VO}_{2 \max }$ e quatro minutos a $70 \%$ do $\mathrm{VO}_{2 \max }$ uma hora por dia, cinco dias por semana) obteve resposta semelhante ao treinamento de moderada intensidade (uma hora por dia a 70\% do $\mathrm{VO}_{2 \max }$ cinco dias por semana) na melhoria da função endotelial mensurada por estimulação de AcH. Este estudo sugere que o uso de treinamento intervalado pode ser eficaz na melhoria das condições de vasodilatação e função endotelial.

Dessa maneira, a vasodilatação induzida pelo exercício aeróbio é importante no tratamento e prevenção da doença arterial-aterosclerótica parece ser mais evidenciada em protocolos que utilizam a intensidade moderada em humanos e animais; porém, evidência recente mostrou que treinamento intervalado de alta intensidade em modelo animal também atua como fator protetor na saúde endotelial.

\section{Exercício físico aeróbio e inflamação}

Conscientes da hipótese de oxidação da LDL no espaço subendotelial, dando início a um processo inflamatório que culminará com a lesão aterosclerótica, torna-se importante a compreensão de como o exercício aeróbio modula a inflamação sistêmica a fim de atenuar o processo aterosclerótico como um todo. A progressão e o desenvolvimento da aterosclerose dependem, em parte, da migração de monócitos para vasos sanguíneos, onde eles se tornam ativos para liberação de citocinas ${ }^{(44)}$.

A concentração plasmática de citocinas é pequena e, em alguns casos, difícil de ser detectada. Este fato acontece porque, geralmente, na circulação sanguínea, essas citocinas desencadeiam funções supressoras do sistema imune, tanto na inflamação excessiva quanto em desordens neuroendócrino-metabólica sistêmica. Dessa maneira, Petersen e Pedersen ${ }^{(45)}$, sugere, que inflamação sistêmica crônica de baixa intensidade está fortemente associada com as doenças crôniconão-transmissíveis, tais como aterosclerose.

As primeiras citocinas na cascata são o fator de necrose tumoral (TNFa) e interleucina 1 (IL1), citocinas pró-inflamatórias e logo após na continuação da cascata vem a interleucina 6 (IL6), tida como pró e anti-inflamatória, seguida da liberação do receptor antagonista de IL1 (IL1ra), receptor antagonista de TNF (sTNF-R) e interleucina 10 (IL10), classificados como fatores anti-inflamatórios ${ }^{(45)}$.

Geralmente, após o exercício agudo, não há aumento das citocinas pró-inflamatórias (IL1 e TNFa). Isto pode ser parcialmente 
explicado pelo aumento de IL6 que induz a síntese dos receptores antagônicos de IL1 e TNFa (IL1 ra e sTNF-R, respectivamente) e, ainda, de outras citocinas anti-inflamatórias como IL10(46) . Porém, independentemente de IL6, o exercício por si só consegue suprimir de outras maneiras a entrada destas citocinas pró-inflamatórias no plasma. Por exemplo, em camundongos Knockout para o gene de IL6, houve modesta diminuição dos níveis plasmáticos de TNFa de repouso após exercício ${ }^{(47)}$. Isto sugere que pode existir dois mecanismos de atenuação de pequenos níveis inflamatórios sistêmicos, um IL6 dependente e outro IL6 independente.

Markovitch et al. ${ }^{(48)}$ não demonstraram aumento, nem de marcadores inflamatórios tais como proteína C reativa (PCR), IL6 e células do sistema imunológico e fatores de agregação plaquetária, nem de fatores antiinflamatórios como IL10, após uma semana de treinamento aeróbio moderado (30 minutos a 50\% do $\mathrm{VO}_{2 \max }$ ) em 12 voluntários sedentários. Este resultado sugere a existência de outro mecanismo na atenuação da inflamação, envolvendo poucas sessões de exercício aeróbio moderado.

Por outro lado, Coppola et al. ${ }^{(49)}$ demonstraram que o exercício físico aeróbio vigoroso (cicloergômetro de pernas, progressivo, até atingir 20 pontos na escala de Borg) agudo, aumentou fatores relacionados à inflamação e agregação plaquetária em voluntários treinados e saudáveis. Porém, cronicamente, Sloan et al.(44) demonstraram que o
Sugere-se que indivíduos com acometimento aterosclerótico não realizem atividade vigorosa, podendo aumentar o risco de inflamação e agregação plaquetária. Entretanto, indivíduos saudáveis podem fazer o uso desta intensidade de exercício, havendo evidências que demonstram que o treinamento diminui o estímulo à liberação de citocinas pró-inflamatórias.

A tabela 1 mostra os principais estudos realizados com diferentes protocolos utilizados.

\section{CONSIDERAÇÕES FINAIS}

De maneira geral, o exercício aeróbio atua tanto na prevenção quanto no tratamento da aterosclerose. Em relação à intensidade do esforço, ainda não há consenso de qual intensidade leva a melhores resultados no processo aterosclerótico. A falta de consenso pode existir pela inexistência de padronização da intensidade do esforço, ora classificando o esforço pelo percentual do $\mathrm{VO}_{2 \text { max }}$ ora pela frequência cardíaca máxima, ora pela frequência cardíaca de reserva. Poucos são os estudos que classificam a intensidade de esforço pela percepção subjetiva de esforço ou pelo limiar anaeróbio e que, são parâmetros que individualizam e dão mais confiabilidade na afirmação de qual o nível de esforço exigido em determinada atividade.

Apesar de diferentes metodologias, algumas evidências parecem

Tabela 1. Estudos de indicadores de aterosclerose em relação à intensidade do exercício aeróbio.

\begin{tabular}{|c|c|c|c|c|}
\hline Primeiro autor e data & Amostra do estudo & $\begin{array}{l}\text { Protocolos de treinamentos utiliza- } \\
\text { dos }\end{array}$ & $\begin{array}{l}\text { Indicador de aterosclerose } \\
\text { utilizado }\end{array}$ & Resultados principais \\
\hline Cadroy $^{(22)}, 2002$ & $\begin{array}{c}\text { Homens, sedentários e sau- } \\
\text { dáveis }\end{array}$ & $\begin{array}{l}\text { Comparação aguda de duas intensida- } \\
\text { des: } 50 \text { ou } 70 \% \text { do } \mathrm{VO}_{2 \max }\end{array}$ & Deposição de plaquetas in vitro & $\begin{array}{c}\text { Grupo que fez a sessão } \\
\text { a } 70 \% \text { aumentou a } \\
\text { tendência de deposição } \\
\text { de plaquetas }\end{array}$ \\
\hline Goto $^{(39)}, 2003$ & Homens jovens e saudáveis & $\begin{array}{c}\text { Comparação crônica (12 semanas) de } \\
\text { três intensidades: } 25 \text { ou } 50 \text { ou } 70 \% \text { do } \\
\qquad \mathrm{VO}_{2 \max }\end{array}$ & $\begin{array}{l}\text { Vasodilatação, pela circulação } \\
\text { sanguínea do antebraço em } \\
\text { resposta à acetil-colina }\end{array}$ & $\begin{array}{l}\text { Somente o grupo que } \\
\text { treinou a 50\% obteve } \\
\text { melhora da função } \\
\text { vasodilatadora }\end{array}$ \\
\hline Wang ${ }^{(33)}, 2006$ & Homens jovens e sedentários & $\begin{array}{l}\text { Comparação aguda de três intensidades: } \\
40 \text { ou } 60 \text { e } 80 \% \text { do } \mathrm{VO}_{2 \max }\end{array}$ & Níveis plasmáticos de LDL-ox** & $\begin{array}{l}\text { Grupo que exercitou a } \\
\text { 80\% teve maiores níveis } \\
\text { de LDL-ox pós-exercício }\end{array}$ \\
\hline Sloan ${ }^{(44)}, 2007$ & Mulheres e homens saudáveis & $\begin{array}{l}\text { Comparação crônica (12 semanas) de } \\
\text { duas intensidades: } 60 \text { ou } 80 \% \text { da } F C_{\max }^{*}\end{array}$ & TNF*** plasmático & $\begin{array}{l}\text { Somente o grupo que } \\
\text { treinou a } 80 \% \text { obteve } \\
\text { melhora na liberação de } \\
\text { TNF pós-exercício }\end{array}$ \\
\hline $\operatorname{Sun}^{(40)}, 2008$ & Ratos Wistar & $\begin{array}{l}\text { Comparação crônica (seis semanas) } \\
\text { de dois protocolos: } 2 \mathrm{~h} \text { ou } 3 \mathrm{~h} \text { por dia de } \\
\text { esteira forçada }\end{array}$ & $\begin{array}{c}\text { Vasodilatação, pela circulação } \\
\text { sanguínea em resposta à acetil- } \\
\text { colina }\end{array}$ & $\begin{array}{c}\text { Somente o grupo que } \\
\text { treinou } 2 \text { h obteve } \\
\text { melhora na função } \\
\text { vasodilatadora }\end{array}$ \\
\hline
\end{tabular}

\footnotetext{
* - Frequência cardíaca máxima, ${ }^{* *}$ - LDL oxidada, ${ }^{* * *}$ - Fator de necrose tumoral.
}

exercício aeróbio de alta intensidade (75 a 80\% da frequência cardíaca máxima, durante 40 minutos, quatro vezes por semana), foi capaz de diminuir $(p<0,05)$ a estimulação de liberação do TNF, ao passo que o exercício aeróbio de moderada intensidade (55 a 60\% da frequência cardíaca máxima, durante 40 minutos, quatro vezes por semana) não conseguiu o mesmo efeito em 12 semanas de treinamento em indivíduos saudáveis, sugerindo, então, que o exercício de alta intensidade crônico pode diminuir a liberação de citocinas pró-inflamatórias como TNF. Neta mesma linha, porém em modelo animal (ratos Wistar), Lana et al. ${ }^{(50)}$, também mostraram que o treinamento aeróbio de alta intensidade (cinco dias por semana, uma hora por dia, $25 \mathrm{~m} / \mathrm{min}$ ) foi mais efetivo na atenuação da resposta inflamatória induzida por carragenina na pata dos animais que o treinamento de baixa intensidade (cinco dias por semana, uma hora por dia, 15m/min). estar claras. De maneira aguda, o exercício físico de alta intensidade leva ao aumento do risco coronariano primário, aumento do estresse oxidativo, diminuição da função vasodilatadora do organismo, aumento da inflamação e agregação plaquetária, sendo, portanto, contraindicado em pessoas previamente acometidas com aterosclerose. Cronicamente, alguns estudos demonstraram melhora e outros, efeitos negativos desses fatores, sendo necessário mais estudos, tanto em humanos quanto em modelos animais, para melhor elucidar o papel da intensidade do treinamento aeróbio na aterosclerose.

Todos os autores declararam não haver qualquer potencial conflito de interesses referente a este artigo. 


\section{REFERÊNCIAS}

1. Instituto Brasileiro de Geografia e Estatística (IBGE). Informações estatísticas e geocientíficas. [citado em 2006 jan 20]. Disponível em http://www.ibge.gov.br.

2. O'Donovan G, Blazevich AJ, Boreham C, Cooper AR, Crank H, Ekelund U, et al. The ABC of Physical Activity for Health: a consensus statement from the British Association of Sport and Exercise Sciences. J Sports Sci 2010;28:573-91.

3. Pinho RA, Araújo MC, Ghisi GL, Benetti M. Coronary heart disease, physical exercise and oxidative stress. Arq Bras Cardiol 2010;94:549-55.

4. Piepoli MF, Corrà U, Benzer W, Bjarnason-Wehrens B, Dendale $\mathrm{P}$, Gaita $\mathrm{D}$, et al. Secondary prevention through cardiac rehabilitation: from knowledge to implementation. A position paper from the Cardiac Rehabilitation Section of the European Association of Cardiovascular Prevention and Rehabilitation. Eur J Cardiovasc Prev Rehabil 2010;17:1-17.

5. Dias RM, Forjaz CL, Cucato GG, Costa LA, Câmara LC, Wolosker N, et al. Obesity decreases time to claudication and delays post-exercise hemodynamic recovery in elderly peripheral arterial disease patients. Gerontology 2009;55:21-6.

6. Brandt C, Pedersen BK. The role of exercise-induced myokines in muscle homeostasis and the defense against chronic diseases. J Biomed Biotechnol 2010;2010:520258.

7. Lippi G, Maffulli N. Biological influence of physical exercise on hemostasis. Semin Thromb Hemost 2009;35:269-76

8. Libby P, DiCarli M, Weissleder R. The vascular biology of atherosclerosis and imaging targets. J Nucl Med 2010;51:33S-375.

9. Barter P. The inflammation: Lipoprotein cycle. Atherosclerosis Supplements 2005;6:15-20.

10. Parthasarathy S, Litvinov D, Selvarajan K, Garelnabi M. Lipid peroxidation and decomposition - conflicting roles in plaque vulnerability and stability. Biochim Biophys Acta 2008;1781:221-31.

11. Yang $H$, Zhou $L$, Wang $Z L$, Roberts $L$, Lin $X$, Zhao $Y$, et al. Overexpression of antioxidant enzymes in ApoE-deficient mice suppresses Benzo(a)pyrene-accelerated atherosclerosis. Atherosclerosis 2009;207:51-8.

12. Asif AR, Hecker M, Cattaruzza M. Disinhibition of SOD-2 Expression to Compensate for a Genetically Determined NO Deficit in Endothelial Cells. Arterioscler Thromb Vasc Biol 2009;29:1890-3.

13. Yin $\mathrm{C}-\mathrm{C}$, Huang $\mathrm{KT}$. $\mathrm{H}_{2} \mathrm{O}_{2}$ but not $\mathrm{O}_{2}^{-}$elevated by oxidized LDL enhances human aortic smooth muscle cell proliferation. J Biomed Sci 2007;14:245-54.

14. Wassmann S, Wassmann K, Nickening G. Modulation of oxidant and antioxidant enzyme expression and function in vascular cells. Hypertension 2004;44:381-6.

15. Lewis P, Stefanovic P, Pete J, Calkin AC, Giunti S, Thallas-Bonke V, et al. Lack of the Antioxidant Enzyme Glutathione Peroxidase-1 Accelerates Atherosclerosis in Diabetic Apolipoprotein E-Deficient Mice. Circulation 2007;115:2178-87.

16. Guo Z, Ran Q, Roberts LJ, Zhou L, Richardson A, Sharan C, et al. Suppression of Atherogenesis by Overexpression of Glutathione Peroxidase-4 in Apolipoprotein E-Deficient Mice. Free Radic Biol Med 2008;44:343-52.

17. Green DJ, Maiorana A, O'Driscoll G, Taylor R. Effect of exercise training on endothelium-derived nitric oxide function in humans. J Physiol 2004;56:1-25

18. Rabêlo LA, Souza VN, Fonseca LJ, Sampaio WO. Redox unbalance: NADPH oxidase as therapeutic target in blood pressure control. Arq Bras Cardiol 2010;94:643-51.

19. Laufs U, Werner N, Link A, Endres M, Wassmann S, Jürgens C, et al. Physical training increases endothelial progenitors cells, inhibits neointima formation, and enhances angiogenesis. Circulation 2004;109:2006-226.

20. Da Silva KS, Nahas MV, Hoefelmann LP, Lopes AS, de Oliveira ES. Associações entre atividade física, índice de massa corporal e comportamentos sedentários em adolescentes. Rev Bras Epidemiol 2008;11:159-68.

21. Cimadon HM, Geremia R, Pellanda LC. Dietary habits and risk factors for atherosclerosis in students from Bento Gonçalves. Arq Bras Cardiol 2010; Jul 9 [Epub ahead of print].

22. Holtermann A, Mortensen OS, Burr H, Søgaard K, Gyntelberg F, Suadicani P. Fitness, work and leisure-time physical activity, and ischaemic heart disease and all-cause mortality among men with pre-existing cardiovascular disease. Scand J Work Environ Health 2010; Mar 29 [Epub ahead of print].

23. Sociedade Brasileira de Cardiologia. IV Brazilian Guideline for Dyslipidemia and Atherosclerosis prevention: Department of Atherosclerosis of Brazilian Society of Cardiology. Arq Bras Cardiol 2007:88 Suppl 1:2-19.

24. Siscovick DS, Weiss NS, Fletcher RH, Lasky T. The incidence of primary cardiac arrest during vigorous exercise. N Engl J Med 1984;311:874-7.

25. Cadroy Y, Pillard F, Sakariassen KS, Thalamas C, Boneu B, Riviere D. Strenuous but not moderate exercise in- creases the thrombotic tendency in healthy sedentary male volunteers. J Appl Physiol 2002;93:829-33.

26. Erikssen G, Liestol K, Bjornholt J, Thaulow E, Sandvik L, Erikssen J. Changes in physical fitness and changes in mortality. Lancet 1998;352:759-62.

27. Kelley GA, Kelley KS. Aerobic exercise and lipids and lipoproteins in children and adolescents: A metaanalysis of randomized controlled trials. Atherosclerosis 2007;191:447-53.

28. Rauramaa R, Halonen P, Vaanen NB, Lakka TA, Arno Schmidt-Trucksa A, Berg A, et al. Effects of Aerobic Physical Exercise on Inflammation and Atherosclerosis in Men: The DNASCO Study. Ann Intern Med 2004;140:1007-14.

29. Shimada KBM, Chiharu Kishimoto MD, Taka-aki Okabe BM, Miki Hattori BM, Toshinori Murayama MD, Masayuki Yokode MD, et al. Exercise Training Reduces Severity of Atherosclerosis in Apolipoprotein E Knockout Mice via Nitric Oxide. Circ J 2007;71:1147-51.

30. Napoli C, Williams-Ignarro S, de Nigris F, Lerman OL, D'Armiento FP, Crimi F, et al. Physical training and metabolic supplementation reduce spontaneous atherosclerotic plaque rupture and prolong survival in hypercholesterolemic mice. PNAS 2006;103:10479-84.

31. Ramachandran S, Penumetcha M, Merchant NK, Santanam N, Rong R, Parthasarathy S. Exercise reduces preexisting atherosclerotic lesions in LDL receptor knock out mice. Atherosclerosis 2005;178:33-8.

32. Williams JK, Kaplan JA, Suparto IH, Fox JL, Manuck, SB. Effects of Exercise on Cardiovascular Outcomes in Monkeys With Risk Factors for Coronary Heart Disease. Arterioscler Thromb Vasc Biol 2003;23:864-71.

33. Pinho RA, Andrades ME, Oliveira MR, Pirola AC, Zago MS, Silveira PC et al. Imbalance in SOD/CAT activities in rat skeletal muscles submitted to treadmill training exercise. Cell Biol Int 2006;30:848-53.

34. Vuorimaa T, Ahotupa M, Irjala K, Vasankari T. Acute Prolonged Exercise Reduces Moderately Oxidized LDL in Healthy Men. Int J Sports Med 2005;26:420-5.

35. Wang JS, Lee L, Chow SE. Role of exercise intensities in oxidized low-density lipoprotein-mediated redox status of monocyte in men. J Appl Physiol 2006;101:740-4.

36. de Moraes C, Davel APC, Rossoni LV, Antunes E, Zanesco A. Exercise training improves relaxation response and SOD-1 expression in aortic and mesenteric rings from high caloric diet-fed rats. BMC Physiology 2008;8:12.

37. Napoli C, Ignarro SW, de Nigris F, Lerman LO, Rossi L, Guarino C, et al. Long-term combined beneficial effects of physical training and metabolic treatment on atherosclerosis in hypercholesterolemic mice. PNAS 2004;101:8797-802.

38. Silva LA, Pinho CA, Rocha LG, Tuon T, Silveira PC, Pinho RA. Effect of different models of physical exercise on oxidative stress markers in mouse liver. Appl Physiol Nutr Metab 2009;34:60-5.

39. Chevion S, Moran DS, Heled Y, Shani Y, Regev G, Abbou B, et al. Plasma antioxidant status and cell injury after severe physical exercise. PNAS 2003;100:5119-23.

40. DeSouza CA, Shapiro LF, Clevenger CM, Dinenno FA, Monahan KD, Tanaka H, et al. Regular Aerobic Exercise Prevents and Restores Age-Related Declines in Endothelium-Dependent Vasodilation in Healthy Men. Circulation 2000;102;1351-7.

41. Goto G, Higashi Y, Kimura M, Noma K, Hara K, Nakagawa K, et al. Effect of Different Intensities of Exercise on Endothelium-Dependent Vasodilation in Humans Role of Endothelium-Dependent Nitric Oxide and Oxidative Stress. Circulation 2003;108:530-5.

42. Sun MW, Zhon MF, Gu F, Qian FL, Gu JC, Chen H. Effects of Different Levels of Exercise Volume on Endothelium-Dependent Vasodilation: Roles of Nitric Oxide Synthase and Heme Oxygenase. Hypertens Res 2008;31:805-16.

43. Haram PM, Kemi OJ, Lee SJ, Bendheim M $\varnothing$, Al-Share QY, Waldum HL, et al. Aerobic interval training vs. continuous moderate exercise in the metabolic syndrome of rats artificially selected for low aerobic capacity. Cardiovasc Res 2009;81:723-32

44. Sloan RP, Shapiro PA, DeMeersman RE, McKinley PS, Tracey KJ, Slavov I, et al. Aerobic exercise attenuates inducible TNF production in humans. J Appl Physiol 2007;103:1007-11.

45. Petersen SM, Pedersen, BK. The anti-inflamatory effect of exercise. J Appl Physol 2005;98:1154-62.

46. Bruunsgrard H. Physical activity modulation of systemic low-level inflammation. J Leukoc Biol 2005;78:819-35.

47. Keller C, Keller P, Giralt M, Hidalgo J, Pedersen BK. Exercise normalizes overexpression of TNF-a in knockout mice. Biochem Biophys Res Commun 2004;321:179-82.

48. Markovitch D, Tyrrell RM, Thompson D. Acute moderate-intensity exercise in middle-aged men has neither an anti- nor proinflammatory effect. J Appl Physiol 2008;105:260-5.

49. Coppola A, Coppola L, Mora LD, Limongelli FM, Grassia A, Mastrolorenzo L, et al. Vigorous exercise acutely changes platelet and B-lymphocyte CD39 expression. J Appl Physiol 2005;98:1414-9.

50. Lana AC, Paulino CA, Gonçalves ID. Efeitos dos Exercícios Físicos Sobre o Edema Inflamatório Agudo em Ratos Wistar. Rev Bras Med Esporte 2008;14:33-7. 